\title{
Rotator Cuff Repair With Knotless Technique Is Quicker and More Cost-Effective Than Knotted Technique
}

\author{
Katherine A. Burns, MD., Lynn Robbins, PA-C., Angela R. LeMarr, RN., BSN., ONC., \\ Amber L. Childress, BS., RN., ONC., Diane J. Morton, MS., and \\ Melissa L. Wilson, MPH., PhD.
}

\begin{abstract}
Purpose: To determine the cost-effectiveness of knotted (KT) versus knotless (KL) methods for rotator cuff surgical repair and to assess differences in patients' outcomes. Methods: We retrospectively identified all patients who underwent arthroscopic rotator cuff repair at 1 institution by 1 surgeon over 2 6-month periods of time (KT technique from August 1 , 2013, through January 31, 2014; and KL technique from December 1, 2014, through May 31, 2015) to calculate the direct and indirect costs associated with arthroscopic KT or KL suture bridge rotator cuff repair. Patient demographics, number of anchors used, tendons repaired, procedure time, operative time, and clinical results were also evaluated. We used univariate generalized linear models with a Gaussian distribution for assessment scores and total and implant cost data. Results: We identified 87 patients for inclusion during the 2 time frames ( $35 \mathrm{KT}, 54 \mathrm{KL}$ ). After excluding patients for tear size $<4 \mathrm{~cm}^{2}(\mathrm{n}=42), \leq 3$ anchors $(\mathrm{n}=5)$, revision surgery $(\mathrm{n}=1)$, and those in whom additional procedures were performed $(n=2)$, 37 eligible subjects remained $\left(\mathrm{n}_{\mathrm{KT}}=15, \mathrm{n}_{\mathrm{KL}}=22\right)$. Median implant costs were statistically significantly higher in the KL group than in the KT group $\left(\mathrm{M}_{\mathrm{KL}}=\$ 2,127, \mathrm{M}_{\mathrm{KT}}=\$ 1,520, \beta=413.7,95 \%\right.$ CI: 242.8, 584.6, $\left.P<.01\right)$, and more anchors were used in the KL group, with KT requiring a median of 4 anchors (IQR: 4, 5) and KL requiring a median of 5 anchors (IQR: 5, 5, $P=.02$ ). Procedure time was cut in half with KL repair (estimated 43.5 minutes) versus KT repair (80 minutes) $(\beta=0.5,95 \% \mathrm{CI}: 0.4,0.6, P<.001)$. Operating room time also was reduced by approximately $40 \%$ (79.5 minutes for KL; 121 minutes for KT $[\beta=0.6, \mathrm{CI}: 0.6,0.7, P<.001])$. Once operating room costs were considered, median costs were found to be significantly lower in the KL group $\left(\mathrm{M}_{\mathrm{KL}}=\$ 3788.40, \mathrm{M}_{\mathrm{KT}}=\$ 4262.90\right.$, $\beta=-492.1,95 \%$ CI: $-840.0,-144.1, P<.01)$. No statistically significant differences were found between groups in mean preoperative, postoperative or postpreoperative differences in the visual analog scale, Simple Shoulder Test, American Shoulder and Elbow Surgeons, or University of California at Los Angeles scores $(P>0.05$ for all). Conclusions: Despite using more anchors and incurring higher implant costs, the KL technique for rotator cuff repair required less surgical procedure time and cost less overall than the KT technique and resulted in equivalent clinical results. Level of Evidence: Level IV Economic and Decision Analyses.
\end{abstract}

\section{Introduction}

$\mathbf{R}$ otator cuff repair is a common orthopedic procedure that has an annual volume estimated to be 200,000 in the United States, and an additional 400,000

From the SSM Health Orthopedics, SSM Health DePaul Hospital (K.A.B., L.R., A.R.L., A.L.C., D.J.M.), St. Louis, Missouri; and Department of Preventive Medicine, University of Southern California (M.L.W.), Los Angeles, California, U.S.A.

The authors report the following potential conflicts of interest or sources of funding: K.A.B. is a consultant for Lima Corporate. Full ICMJE author disclosure forms are available for this article online, as supplementary material.

Disclosures: Nothing of value was received by any author for the conduction or publication of this study. SSM Health Institutional Review Board. Approved, Study \#15-11-0763. people have surgery related to rotator cuff tendonitis and partial tears. Rotator cuff repair has been shown to significantly improve quality of life. ${ }^{1}$ As arthroscopic techniques and implants continue to evolve, the use of

\footnotetext{
Received April 12, 2019; accepted September 11, 2019.

Address correspondence to Katherine A. Burns, MD, SSM Health Orthopedics, 12349 DePaul Drive, Suite 100, Bridgeton, MO 63044, U.S.A. E-mail: katherine.a.burns.md@ssmhealth.com

(C) 2019 by the Arthroscopy Association of North America. Published by Elsevier Inc. This is an open access article under the CC BY-NC-ND license (http://creativecommons.org/licenses/by-nc-nd/4.0/).

2666-061X/19483

https://doi.org/10.1016/j.asmr.2019.09.005
} 
newer implants requires technical, clinical and cost considerations. Suture anchors represent the most expensive and variable surgeon-directed cost in rotator cuff repair. ${ }^{2}$ In light of increasing individual and institutional costs for health care, a need exists to consider the economic viability of newer implants and techniques.

The primary objective of the study was to determine the cost-effectiveness of knotted (KT) versus knotless (KL) methods of rotator cuff surgical repair and to assess any differences in patients' outcomes. A secondary objective of the study was to compare total variable costs, specifically implant costs and operating room time costs, associated with KT versus KL rotator cuff repair. Our hypothesis was that a decrease in operating room time for cases repaired with KL technique would result in overall cost savings for the same procedure, despite the increased cost of implants. We also hypothesized that clinical outcomes would be similar in the 2 groups.

\section{Methods}

We conducted a retrospective cohort study with institutional review board approval. All patients undergoing arthroscopic rotator cuff repair performed by a single surgeon at a single institution were selected from 2 different 6-month periods of time. Both techniques had been used for a minimum of 10 months before the collection period to minimize any learning-curve effects.

Inclusion criteria included all patients who underwent arthroscopic repair during the 2 time frames selected. Exclusion criteria included tear size less than $4 \mathrm{~cm}^{2}$, fewer than 4 anchors used, revision surgery, and concurrent associated procedures that could prolong operative time, such as distal clavicle excision. All patients reported shoulder pain and had a documented full or substantial partial thickness rotator cuff tear.

\section{Data Collection}

Data were obtained via secure reviews of the electronic medical records and included reviews of the operative notes, operative records, anesthesia records, and implant logs. Preoperative data collection included basic demographics, clinical histories and presurgery questionnaires to evaluate pain and symptoms, including a visual analog scale of 0-10 for pain (VAS), the Simple Shoulder Test (SST), the American Shoulder and Elbow Surgeons (ASES) score, and the University of California at Los Angeles (UCLA) score. These questionnaires were scanned into the electronic medical records and entered into a database. Surgical data collected included the American Society of Anesthesiologists status, operating room time, procedure time, and number and type of implants. Procedure time was defined from the minute of incision to the procedure's end. Operating room time was defined as the total time the patient was in the operating room and included time for induction and extubation. Pre- and postoperative data collection included evaluations of the VAS, SST, ASES, and UCLA questionnaires prior to surgery and again at a median of 10 months postoperatively.

\section{Surgical Technique}

Arthroscopic repair was performed in the lateral decubitus position using interscalene block and general anesthesia. Ten pounds of axial traction were applied for the duration of the case. The operative arm was sterilely prepped and draped. The shoulder joint was inspected arthroscopically and the tear pattern assessed. Tear size and tendon involvement were determined by using the probe and arthroscopic visualization. Four portals were routinely used for tears $4 \mathrm{~cm}$ and larger.

All patients received subacromial decompression and treatment of the biceps as deemed appropriate at the time of surgery (no treatment, tenodesis or tenotomy). The rotator cuff was mobilized, and the cuff footprint was prepared with a shaver and cautery. The tear then was repaired by using a suture bridge technique with either knotted anchors (Smith and Nephew, Healicoil double-loaded anchors and Footprint) or knotless anchors (Arthrex, Speedbridge and Swivelock).

For KT repair, 2 anchors were placed medially, and each limb of the suture was passed and retrieved. Knots were tied to create a mattress stitch medially. One limb of the tied sutures from each anchor then was secured into a lateral row anchor to create a suture bridge repair. This type of repair involved a minimum of 4 passes, 4 suture retrievals, 2 knots tied, and 4 anchors.

For KL repair, 2 anchors were placed medially and 1 pass of 2 swedged sutures was performed. Retrieval was not necessary because a self-retrieving device was used. The swedged sutures then were cut and separated. One limb from each anchor was incorporated into the lateral row. Additional looped sutures (Fiberlink, Arthrex) were passed and incorporated into the lateral row as necessary. This type of repair required a minimum of 2 passes, no suture retrievals, no knots tied, and 4 anchors.

\section{Cost Calculations}

Equivalent variable costs for the 2 groups were not included in cost calculations. Costs not included were surgeons' fees, anesthesia charges (including charges related to receiving interscalene blocks), physicianassistant fees, and physical therapy fees. Imaging fees also were not included because all patients were assumed to have received 1 MRI preoperatively. Indirect costs, including absence from work, lost income, disability, and other social costs were not calculated and 


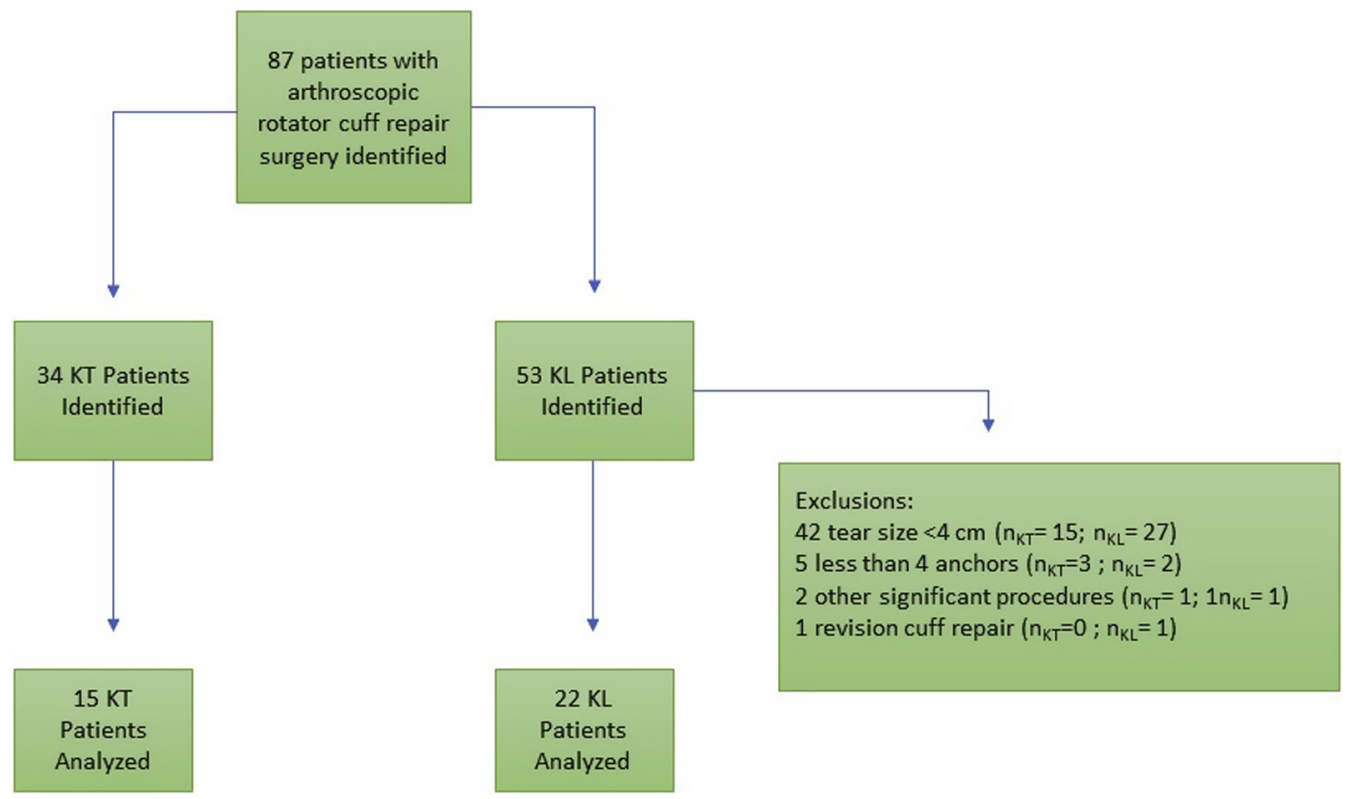

Fig. 1. STROBE diagram showing patient selection and study flow. The source patient population was divided into the 2 surgical technique groups, knotted versus knotless. After exclusions for predefined exclusion criteria, the proportions of patients selected for the study population from the source population were $42.9 \%$ for the knotted group and $40.7 \%$ for the knotless group.

were assumed to be the same in both groups. No adjustments for cost-of-living or other inflationary costs during the 2 time periods were made because the yearto-year inflation rate in the United States was less than 1\% between August 2013 and May 2015 (Consumer Price Index inflation calculator, Bureau of Labor Statistics, United States Department of Labor).

Direct variable cost differences between repair types resulted directly from cost differences in the implants. Cost for the knotless repair included 4 anchors in a kit $(\$ 1,660)$, with an additional $\$ 467$ for each additional anchor and suture in the patients who received knotless subscapularis repair. Cost for the knotted repair was $\$ 1,520$ for 4 anchors, with an additional $\$ 360$ added for each additional anchor in patients who underwent subscapularis repair. These numbers reflect the institutional costs, not the retail price of the implants.

After careful review of individual items, the costs of other disposable equipment used in standard shoulder arthroscopy were determined to be substantially equivalent. They included drapes, arm-suspension devices, pump tubing and suctions, electrocautery and shaver, suture materials for skin closure, dressing, and sling. The cost of the disposable needle was slightly different (\$193 per case with KT repair, \$170 per case with KL) as was the cost of 1 cannula ( $\$ 31.20$ per case in $\mathrm{KT}$ vs $\$ 27.50$ in $\mathrm{KL}$ ). These cost differences were negligible and were not included in cost calculations.

Table 1. Characteristics of Study Subjects by Knotted or Knotless Technique

\begin{tabular}{|c|c|c|c|}
\hline Characteristic $^{*}$ & $\mathrm{KT}(\mathrm{n}=15)$ & $\mathrm{KL}(\mathrm{n}=22)$ & $P$ Value ${ }^{\dagger}$ \\
\hline Age, years & $61.6 \pm 9.1$ & $63.2 \pm 9.7$ & 0.61 \\
\hline Female sex & $9(60)$ & $13(62)$ & 0.58 \\
\hline Body mass index, $\mathrm{kg} / \mathrm{m}^{2}$ & $33.6 \pm 9.0$ & $31.9 \pm 5.8$ & 0.49 \\
\hline Right shoulder & $8(53)$ & $15(68)$ & 0.49 \\
\hline $\mathrm{ASA}^{\ddagger \S}$ & $2(2,3)$ & $2(2,3)$ & 0.87 \\
\hline Diabetes & $4(27)$ & $9(41)$ & 0.49 \\
\hline Estimated tear size, $\mathrm{cm}^{2}$ & $8.3 \pm 4.6$ & $9.8 \pm 6.7$ & 0.55 \\
\hline Number of anchors ${ }^{\S}$ & $4(4,5)$ & $5(5,5)$ & 0.02 \\
\hline Follow-up time (months) & $30(28,30)$ & $13.5(11.5,15.5)$ & $<0.001$ \\
\hline
\end{tabular}

KT, knotted; KL, knotless.

*Presented as (mean \pm standard deviation) or as count (\%), except where noted.

${ }^{\dagger} P$ values obtained by $t$ tests or Wilcoxon signed rank tests for continuous or ordinal variables or Fisher exact tests for categorical variables.

${ }^{\ddagger}$ American Society of Anesthesiologists classification.

${ }^{\S}$ Presented as median (interquartile range). 


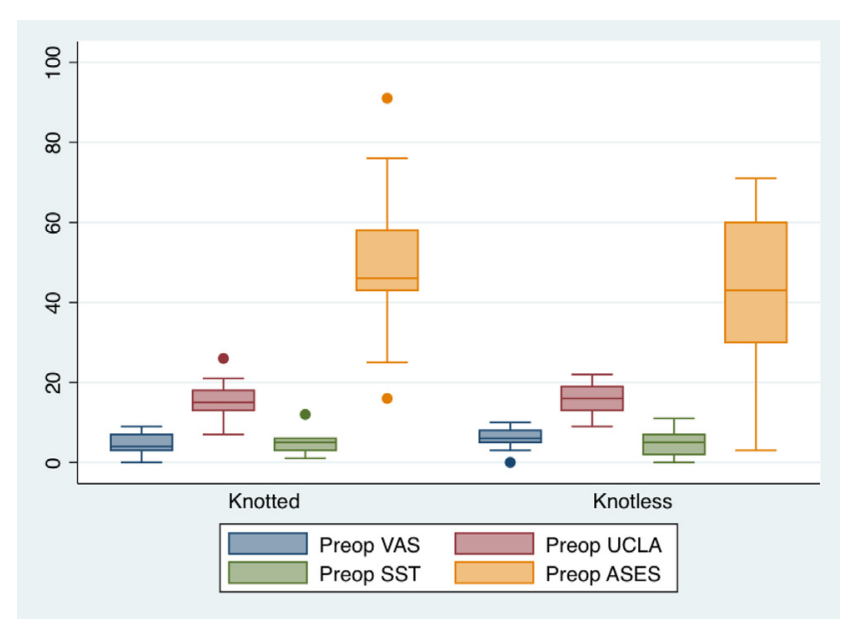

Fig. 2. A box plot of median preoperative patient outcome scores for knotted versus knotless rotator cuff repair. The middle lines represent median values, and the boxes represent the lower and upper quartiles of the outcome score being plotted. The vertical lines represent the upper and lower adjacent values (UAV and LAV), and the dots represent values outside of the adjacent values. The UAV are defined as the largest observation that is less than or equal to the third quartile (Q3) plus 1.5 times the interquartile range (IQR). Similarly, the LAV are the smallest observations that are greater than or equal to the first quartile $(\mathrm{Q} 1)$ minus $1.5 * \mathrm{IQR}$. Results suggest no differences at baseline between groups for any of the patient-outcome scores.

Indirect variable cost differences between repair types resulted directly from differences in procedure time and operating room time. The cost of operating room time was determined using our institution's calculated cost per minute based on the level of the case. Variable costs per minute of operating room time at our institution ranged from $\$ 15.73$ per minute to $\$ 42.87$ per minute, with an average of $\$ 28.20$ per minute. For the purpose of this study, a rate of $\$ 22.30$ per minute was used, reflecting the most commonly used estimate for orthopedic cases and based on the complexity of a standard arthroscopic rotator cuff repair.

Time-dependent costs, including increased use of arthroscopy fluids in longer cases, were attributed to the cost per minute of operative time and were not independently evaluated. Operating room staffing was the same for the 2 procedures; any differences in operating room staffing costs were assumed to be a product of operating room time. Indirect costs with regard to increased or decreased surgeon productivity and the value of the surgeon's time in the operating room also were not calculated. Total variable costs for the purpose of this study included only the costs determined to be substantially different between the 2 procedures, with a direct component (implant cost) and an indirect component (operating room time and procedure time).

\section{Statistical Analysis}

Cost data were analyzed preliminarily using the Wilcoxon rank-sum (Mann-Whitney) test. Demographic and clinical characteristics were compared between KT and KL techniques using Student $t$ tests for normally distributed, continuous variables and using the Wilcoxon rank sum test for nonparametric continuous variables. A Fisher exact test was used for categorical variables. To evaluate differences in pre- and postoperative assessment scores as well as total and implant cost data, we used univariate generalized linear models with a Gaussian distribution. We modeled the relationship between operative and procedure times and surgical technique using generalized linear models with a gamma distribution. We considered several a priori factors to be potential confounders: the number of anchors used, whether repair of the subscapularis was required and whether biceps tenodesis or tenotomy was performed as an associated procedure. Only the number of anchors altered the effect estimate by more than 15\% (and only in the time models) and was included in the final models. Neither subscapularis repair nor treatment of the biceps was associated with the outcome, and they were not confounders; thus, they were not included in the final model. Model fit was evaluated by inspection of residuals via q-normal plots. Statistical significance was set at $\propto=.05$, and no adjustments were made for multiple comparisons. Statistical analysis was performed using Stata 14.0 (Stata Corp, College Station, Texas).

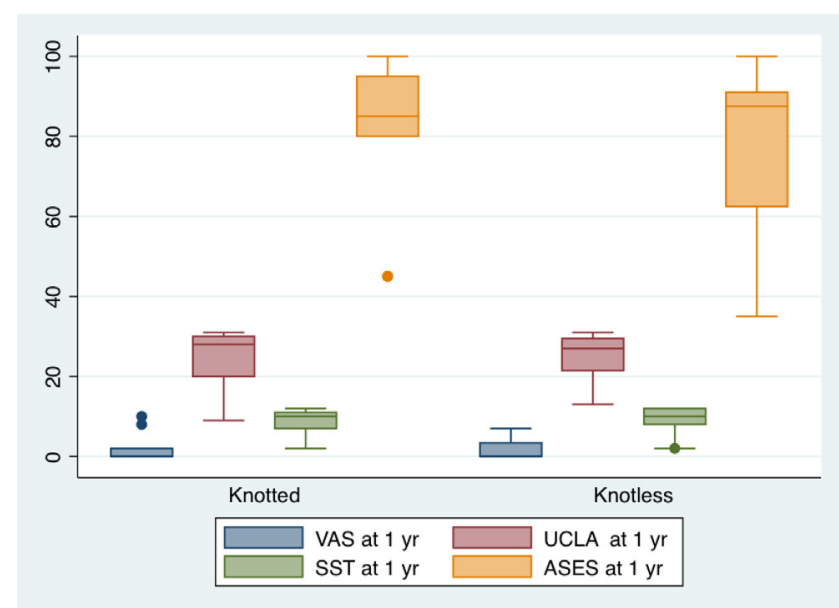

Fig. 3. A box plot of median postoperative (minimum 1 year) patient outcome scores for knotted versus knotless rotator cuff repair. Middle lines represent median values; boxes represent lower and upper quartiles of the outcome score being plotted; vertical lines represent the upper and lower adjacent values (UAV and LAV); dots represent values outside of the adjacent values. UAV, largest observation $\leq \mathrm{Q} 3+1.5 * \mathrm{IQR} ; \mathrm{LAV}$, smallest observation $>=$ Q1-1.5*IQR. Results suggest no differences at postoperative assessment between groups for any of the patient-outcome scores. 
Table 2. Preoperative and Postoperative Assessment Scores of Study Subjects After Knotted or Knotless Technique

\begin{tabular}{|c|c|c|c|c|c|c|}
\hline Variable & Knotted (N) & KT Score & Knotless (N) & KL Score & $\beta(95 \% \mathrm{CI})$ & $P$ value ${ }^{\dagger}$ \\
\hline \multicolumn{7}{|l|}{ Preoperative } \\
\hline VAS & 15 & $4.7 \pm 2.9$ & 22 & $6.2 \pm 2.6$ & $1.4(-0.4,3.2)$ & 0.12 \\
\hline SST & 15 & $4.9 \pm 2.4$ & 22 & $4.7 \pm 2.8$ & $-0.1(-1.9,1.6)$ & 0.88 \\
\hline UCLA & 15 & $15.5 \pm 4.4$ & 22 & $15.5 \pm 4.1$ & $9.5 \mathrm{e}-3(-2.8,2.8)$ & 0.99 \\
\hline \multicolumn{7}{|l|}{ Postoperative } \\
\hline VAS & 11 & $1.8 \pm 3.6$ & 16 & $1.6 \pm 2.3$ & $-0.3(-2.5,2.0)$ & 0.81 \\
\hline UCLA & 11 & $24.4 \pm 7.3$ & 16 & $24.8 \pm 6.1$ & $0.4(-4.6,5.5)$ & 0.86 \\
\hline \multicolumn{7}{|c|}{ Postoperative-preoperative } \\
\hline$\Delta$ VAS & 11 & $3.0 \pm 3.2$ & 16 & $4.4 \pm 2.1$ & $1.4(-0.6,3.4)$ & 0.17 \\
\hline$\Delta \mathrm{SST}$ & 11 & $4.6 \pm 3.9$ & 16 & $4.7 \pm 2.7$ & $0.1(-2.5,2.6)$ & 0.97 \\
\hline$\triangle \mathrm{ASES}$ & 11 & $34.0 \pm 19.2$ & 16 & $35.7 \pm 19.9$ & $1.7(-13.4,16.8)$ & 0.83 \\
\hline$\Delta \mathrm{UCLA}$ & 11 & $9.5 \pm 8.6$ & 16 & $8.8 \pm 5.5$ & $-0.7(-6.1,4.8)$ & 0.81 \\
\hline
\end{tabular}

No a priori power analysis was conducted because the number of patients was fixed. However, a post hoc power analysis with the achieved sample size demonstrated $79 \%$ power to detect an effect size of $\mathrm{f}^{2}=0.22$ (equivalent to $\mathrm{R}^{2}=0.18$, the observed effect size in this study), assuming a 2 -sided $\alpha=0.05$ and 1 predictor.

\section{Results}

The KT technique cohort included 35 patients who underwent rotator cuff surgery with the KT technique between August 1, 2013, and January 31, 2014. An additional 54 patients underwent rotator cuff repair surgery with the KL technique between December 1 , 2014, and May 31, 2015, for a total of 87 patients identified. Patients were excluded for tear size $<4 \mathrm{~cm}^{2}$ $(n=42)$, fewer than 4 anchors $(n=5)$, revision surgery $(\mathrm{n}=1)$, and miniopen repairs $(\mathrm{n}=2)$. Thirty-seven patients ( $\mathrm{n}=15 \mathrm{KT}, \mathrm{n}=22 \mathrm{KL}$ ) met all criteria and were included in the study population (Fig. 1). Characteristics of the study population by technique are summarized in Table 1. No statistically significant differences existed in the 2 groups at baseline with regard to age, sex, smoking status, body mass index, percentage of right shoulders represented, American Society of Anesthesiologists scores, and diagnosis of diabetes or hypertension $(P>.05$ for all).

Of the patients, $11(73 \%)$ in the KT group and 16 $(73 \%)$ in the KL group had complete follow-up data available for review. Median follow-up time was 30 months (IQR: 28, 30) for patients who received KT repair and 13.5 (IQR: 11.5, 15.5) for those who received $\mathrm{KL}$ repair $(P<.001)$. The difference in follow-up time reflects the difference in the time periods over which the data were collected; KT repairs were performed approximately 1 year before KL repairs.

Groups did not differ with respect to clinical assessment scores (Fig. 2) (Fig. 3), either preoperatively or at time of last follow-up. Specifically, no differences occurred in mean preoperative or postoperative VAS, SST, ASES, or UCLA scores $(P>.05$ for all) (Table 2). Additionally, both groups reported improvement from pre- to postsurgery for all selfreported patient scores $(P<.01$ for all) (data not shown), but there were no differences in postoperative, preoperative or postpreoperative scores between groups $(P>.05$ for all).

Table 3. Direct and Variable Costs for Knotted and Knotless Technique

\begin{tabular}{|c|c|c|c|c|}
\hline Variable $^{*}$ & Knotted $(\mathrm{n}=15)$ & Knotless $(n=22)$ & $\beta(95 \% \mathrm{CI})^{\dagger}$ & $P$ value ${ }^{\dagger}$ \\
\hline$\overline{\text { Direct cost (implant cost) }}$ & $\$ 1,520(1,520,1,880)$ & $\$ 2,127(2,127,2,127)$ & $413.7(242.8,584.6)$ & $<0.01$ \\
\hline Total variable $\operatorname{cost}^{\ddagger}$ & $\$ 4,262.9(3,973,4,823.6)$ & $\$ 3,788.4(3,621.1,4,044.8)$ & $-492.1(-840.0,-144.1)$ & $<0.01$ \\
\hline
\end{tabular}

*Presented as median (interquartile range).

${ }^{\dagger}$ Betas and $P$ values obtained by generalized linear models with a Gaussian distribution. Beta can be interpreted as the expected mean change in cost for knotless compared to knotted technique.

${ }^{\ddagger}$ Implant cost plus operating room time cost. 
Table 4. Operating Room Time and Procedure Time for Knotted vs Knotless Rotator Cuff Repair

\begin{tabular}{|c|c|c|c|c|}
\hline Variabl $^{*}$ & Knotted $(\mathrm{n}=15)$ & Knotless $(\mathrm{n}=22)$ & $\operatorname{Exp}(\beta)(95 \% \mathrm{CI})^{\dagger}$ & $P$ Value \\
\hline \multicolumn{5}{|l|}{ Time (minutes) } \\
\hline Operating room & $121(109,137)$ & $79.5(68,88)$ & $0.6(0.6,0.7)$ & $<0.001$ \\
\hline Procedure & $80(66,90)$ & $43.5(37,48)$ & $0.5(0.4,0.6)$ & $<0.001$ \\
\hline
\end{tabular}

*Presented as median (interquartile range).

${ }^{\dagger}$ Betas and $P$ values were obtained using generalized linear models with a gamma distribution, adjusted for the number of anchors. Betas are reported as $\exp (\beta)$ and can be interpreted as the ratio of the mean times for the knotless (KL) procedure to the knotted (KT) procedure, adjusted for the number of anchors used. Thus, numbers below 1 indicate a lower operating/procedure time in the KL group relative to the KT group.

Median implant costs were higher in the KL group $(\$ 2,127$ for KL, $\$ 1,520$ for KT, $P<.001)$ (Table 3). The number of anchors used differed between KT and KL repair, with KT requiring a median of 4 anchors (IQR: $4,5)$ and KL requiring a median of 5 anchors (IQR: 5, 5, $P=.02$ ). Repair of the subscapularis also was different between groups; $40 \%$ of the KT population had this procedure versus $77.3 \%$ of the KL group $(P=.04)$.

Both median operating room time and median procedure time were statistically significantly longer for the KT than for the KL procedure after adjusting for the number of anchors (Table 4). The KT technique required an estimated 80 minutes (IQR: 66, 90), whereas the time was approximately half that for the KL technique $(\mathrm{M}=$ 43.5, IQR: 37,$48 ; \beta=0.5, P<.001)$. Likewise, the median operative time for the KL technique was statistically significantly less than that of the KT technique, reducing the time by about $40 \%(\beta=0.6, P<.001)$ to 79.5 minutes (IQR: 68, 88) compared with 121 minutes (IQR: 109, 137) for the KT group.

Evaluation of the difference in total variable costs between the procedures (implant costs and operating room time costs) resulted in median costs of $\$ 3,788.35$ for $\mathrm{KL}$ and $\$ 4,262.90$ for KT. KL repair was significantly less costly when incorporating time savings $(P<.01)$ (Table 3).

\section{Discussion}

We found that KL suture bridge rotator cuff repair, overall, was faster and less costly than KT suture bridge repair, despite higher implant cost. Clinical results for these 2 types of procedures were equivalent at 1-year follow-up. Our study looked specifically at implant costs because these direct costs may negatively impact the cost-effectiveness of rotator cuff repair. Delivering value to the patient in rotator cuff repair requires evaluation of cost while maintaining good patient outcomes.

Vitale et al. found that the cost-effectiveness for rotator cuff repair compared favorably to other orthopedic procedures. ${ }^{1}$ In their analysis, implant costs were not directly evaluated and were included in operating room costs. A recent review found that implants represented the most variable and most expensive of surgeon-directed cost in rotator cuff repair. ${ }^{2}$ Newer implant designs may carry a price premium and may directly affect the cost and, potentially, the cost-effectiveness of rotator cuff repair.

The direct costs of implants have been examined previously. Black et al. also reviewed the effect of direct implant costs by comparing transosseous equivalent and anchorless tunnel technique for rotator cuff repair. They found cost savings in avoiding the use of anchors without a significant effect on operative time. ${ }^{3}$ This cost differential increased with increasing numbers of anchors. They also found evidence of a learning curve, with first cases requiring significantly more time than later cases. The learning-curve effect was minimized by selecting a 6-month window after a minimum of 10 months' use of the implant system. Our study also confirmed that increasing number of implants increases direct costs; however, this was more than offset by operating room time savings. Tear-size effect was controlled in our study by excluding patients with tear sizes $<4 \mathrm{~cm}^{2}$.

Time savings can add significant value, especially in ambulatory outpatient surgical centers and should be considered in cost analysis. Shorter operative times may carry additional benefits for the patient because shorter operative times were found to be predictive of less pain at rest. ${ }^{4}$ Patients also may experience fewer side effects of anesthesia, such as nausea, vomiting and somnolence. For the purposes of this study, postoperative pain management was assumed to be equivalent for both groups; any cost reduction related to reduced pain management needs for shorter operative times in this study would favor the KL group.

Strengths of the current study include the fact that the study was conducted over a relatively short time, limiting the changes in costs that would occur due to normal fluctuations in the economy. Additionally, because all surgeries were performed by 1 surgeon, little variability in surgical or postoperative protocol could be attributed throughout the course of the study. Both time periods were selected to limit any learning-curve effect; there was a minimum of 10 months of use for each technique. Although follow-up times varied by group, due to the differing time periods in which the procedures were performed, any variance in cost of living as well as small variances in the cost of cannulas and needles were considered negligible. The KL group procedures were performed primarily in 2015 , compared with the KT 
group procedures performed in 2013, so any cost variations would favor KT over KL because costs would be expected to rise over time.

Rotator cuff repair continues to be 1 of the most commonly performed orthopedic procedures, and these types of routine orthopedic procedures will come under increasing scrutiny to limit cost and increase value. Although the cost of implants continues to receive attention from both health care institutions and insurers, the value of 1 given implant or surgical technique over another must account for indirect costs, including operative time, while maintaining good clinical outcomes.

As health care spending is more closely examined at a national level, close analysis of efficiency (cost/procedure) and value (cost/benefit) is necessary to justify basic orthopaedic procedures such as rotator cuff repair. Direct costs are only 1 aspect of health care expenditure, and weighing indirect benefits, including time savings and clinical outcomes, including retear rate and quality-of-life years, may affect perceived value.

\section{Limitations}

A significant limitation of this study was the small sample size, increasing the likelihood of type II error. Nevertheless, the differences in operative time, procedure time and cost savings were large and detectable with this sample size, though we cannot rule out that we may have missed differences in VAS, SST, ASES, or UCLA scores.

A limitation of this study was that any differences in retear rates between the 2 methods were not evaluated. The cost-effectiveness of rotator cuff repair may be affected by the relative risk of retear rates because revision surgery for retear carries an additional cost burden. ${ }^{6}$ Bisson calculated the costs to the US healthcare system to transition from the single row to the suture bridge technique and found that large decreases in revision rates would be necessary to justify the added cost. $^{7}$ Brown et al. performed a meta-analysis to evaluate suture configuration, repair method and tear size on retear rates and found that the suture technique may not affect rerupture rates after rotator cuff repair. ${ }^{8}$ A more recent prospective comparative study found lower retear rates in a conventional $\mathrm{KT}$ technique as compared to a KL technique, although the difference was not statistically significant. ${ }^{9}$ This study did not account for biomechanical differences in the 2 techniques; there is evidence to suggest that tying the medial row may allow for better footprint apposition and better initial biomechanical strength than the KL technique. ${ }^{10}$ The incidence of retear in this population is not known because routine postoperative imaging of the repaired rotator cuff was not performed.

The median follow-up time of 10 months was relatively short. Thus, it is possible that over time (due to retear rates and other clinical factors), the cost differential between the 2 techniques may alter. This possibility requires additional evaluation in future studies.

Another limitation includes the retrospective nature of the data collection, which limits the availability of information because it was not collected specifically for the purposes of this research study. For example, it would be beneficial to include an assessment of quality of added life years as well as an assessment of societal costs which, had the study been conducted prospectively, would have been collected.

A third possible limitation includes the potential for misclassification on the basis of tear size because this variable was estimated at the time of surgery and not measured directly. However, previous research has shown that intraoperative measurement does correlate well with actual tear size. ${ }^{5}$ Furthermore, misclassification on the basis of tear size would be expected to be nondifferential with respect to surgical technique and, thus, would tend to bias results toward the null. Another potential limitation regarding tear size was the selection only of tears larger than $4 \mathrm{~cm}^{2}$ and requiring 4 or more anchors; these criteria limit the generalizability of our results to these types of repairs.

Another limitation is potential for transfer bias, in that patients who continued with follow-up may have been different from those who were lost to follow-up. Specifically, if those who remain in the study are more or less likely to return for follow-up, and this differs by study group, the effect observed in the study may be different from the effects that would have been obtained in the whole sample. The surgeon used only 1 repair technique during each of the 2 time points, so selection bias with respect to patient characteristics would be minimized.

\section{Conclusions}

Despite using more anchors and incurring higher implant costs, the KL technique for rotator cuff repair required less surgical procedure time and cost less overall than the KT technique, while resulting in equivalent clinical results.

\section{References}

1. Vitale MA, Vitale MG, Zivin JG, Braman JP, Bigliana LU, Flatow EL. Rotator cuff repair: An analysis of utility scores and cost-effectiveness. J Shoulder Elbow Surg 2007;16: $181-187$.

2. Terhune EB, Cannamela PC, Johnson JS, et al. Surgeondirected cost variation in isolated rotator cuff repair. Orthop J Sports Med 2016;12:1-5.

3. Black EM, Austin LS, Narzikul A, Seidl AJ, Martens K, Lazarus MD. Comparison of implant cost and surgical time in arthroscopic transosseous and transosseous equivalent rotator cuff repair. J Shoulder Elbow Surg 2016;25:1449-1456. 
4. Walton J, Murrell GA. Effects of operative time on outcomes of rotator cuff repair: A two-year clinical outcomes study of 400 patients, comparing open surgery and arthroscopy for rotator cuff repair. Bone Joint Res 2012;1: 210-217.

5. Bryant L, Shnier R, Bryant C, Murrell GA. A comparison of clinical estimation, ultrasonography, magnetic resonance imaging, and arthroscopy in determining the size of rotator cuff tears. J Shoulder Elbow Surg 2002;1 1:219-224.

6. Huang AL, Thavorn K, van Katwyk S, MacDonald P, Lapner P. Double-row arthroscopic rotator cuff repair is more cost-effective than single-row repair. J Bone Joint Surg Am 2017;99:1730-1736.

7. Bisson L, Zivaljevic N, Sanders S, Pula D. A cost analysis of single-row versus double-row and suture bridge rotator cuff repair methods. Knee Surg, Sports Traumatol, Arthrose 2015;23:487-493.

8. Brown MJ, Pula DA, Kluczynski MA, Mashtare T, Bisson LJ. Does suture technique affect re-rupture in arthroscopic rotator cuff repair? A meta-analysis. Arthroscopy 2015;31:1576-1582.

9. Kim KC, Shin HD, Lee WY, Yeon KW, Han SC. Clinical outcomes and repair integrity of arthroscopic rotator cuff repair using suture-bridge technique with or without medial tying: prospective comparative study. J Orthop Surg Res 2018;13:212.

10. Mall NA, Lee AS, Chahal J, et al. Transosseous-equivalent rotator cuff repair: A systematic review on the biomechanical importance of typing the medial row. Arthroscopy 2013;29:377-386. 